\title{
Desmoid Tumor of the Pelvis in Children: One Case Report
}

\author{
Hana El Mansouri ${ }^{1}$, Amal Zourair ${ }^{1}$, Hanane Hammaoui ${ }^{1}$, Salma Amouzoune ${ }^{2}$, \\ Ibtissam Zouita1, Dounia Basraoui' ${ }^{1}$, Hanane Rais ${ }^{2}$, Hicham Jalal ${ }^{1}$ \\ ${ }^{1}$ Department of Radiology of Mother and Child Hospital, Med VI University Hospital, Marrakesh, Morocco \\ ${ }^{2}$ Department of Anatomic Pathology, Med VI University Hospital, Marrakesh, Morocco \\ Email: hana.elmansouri@gmail.com
}

How to cite this paper: El Mansouri, H., Zourair, A., Hammaoui, H., Amouzoune, S., Zouita, I., Basraoui, D., Rais, H. and Jalal, H. (2019) Desmoid Tumor of the Pelvis in Children: One Case Report. Open Access Library Journal, 6: e5122.

https://doi.org/10.4236/oalib.1105122

Received: December 14, 2018

Accepted: January 7, 2019

Published: January 10, 2019

Copyright $\odot 2019$ by author(s) and Open Access Library Inc.

This work is licensed under the Creative Commons Attribution International License (CC BY 4.0).

http://creativecommons.org/licenses/by/4.0/

\begin{abstract}
Desmoid tumors are invasive and aggressive deep fibromatosis rare in children. They occur in muscles, their sheaths and fasciae. Medical imaging (CT and MRI) can suggest the diagnosis, guide the therapeutic management and the follow-up. We report a pediatric case of desmoid tumor of the pelvis through which we describe the clinical and radiological appearance of these tumors in children.
\end{abstract}

\section{Subject Areas \\ Radiology, Medical Imaging}

\section{Keywords}

Desmoid Tumor, Pelvic, Children, Imaging

\section{Introduction}

Desmoid tumors are aggressive, deep fibromatosis, rare in children, they occur in muscles, their sheaths and fasciae [1] [2]. Medical imaging (CT and MRI) can suggest the diagnosis, guide the therapeutic management and the follow-up. We report a pediatric case of desmoid tumor of the pelvis through which we describe the clinical and radiological appearance of these tumors in children.

\section{Patient and Observation}

Our patient is an 8-year-old child, with no particular medical history, admitted for pelvic mass, pain of the rights iliac fossa and the flank of progressive onset. The clinical examination found a painless pelvic mass fixed on the deep tissue and a sensitivity of the right flank. 
Ultrasonography, performed firstly, showed a large pelvic mass lateralized toward the right, ill-defined, of heterogeneous echogenicity, vascularized at Color Doppler Imaging in places, associated with a moderate dilatation of the right excretory cavities.

In CT the tumor appeared quite well limited, measuring approximately $12 \times 7$ $\mathrm{cm}$, isodense compared to muscle, heterogeneous, enhanced by the contrast, adherent to the digestive loops and the right external iliac vessels. It strangles the right ureter, also infiltrates the transverse muscle, compresses and represses nearby structures, especially the bladder (Figure 1 and Figure 2).

A biopsy of the mass was performed. Histopathologic examination yielded a diagnosis of desmoid tumor. The child was put under chemotherapy before possible surgical treatment.
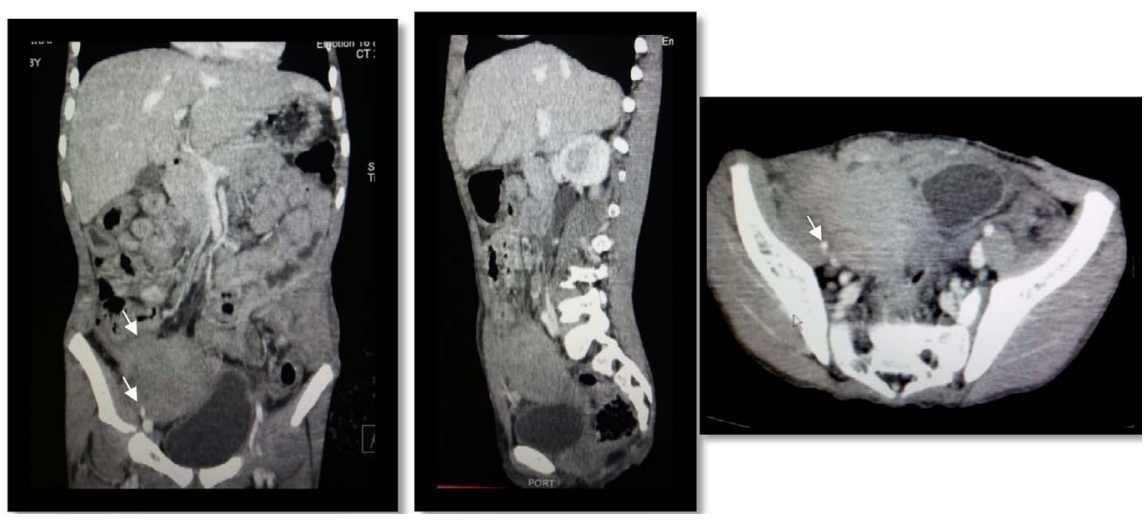

Figure 1. Abdominal CT with contrast. Coronal, sagital and axial reconstruction images showing: Large heterogeneous pelvic mass, isodense to muscle, adherent to the digestive loops and external iliac vessels (arrow). It infiltrates the transverse muscle and compresses and represses the bladder.
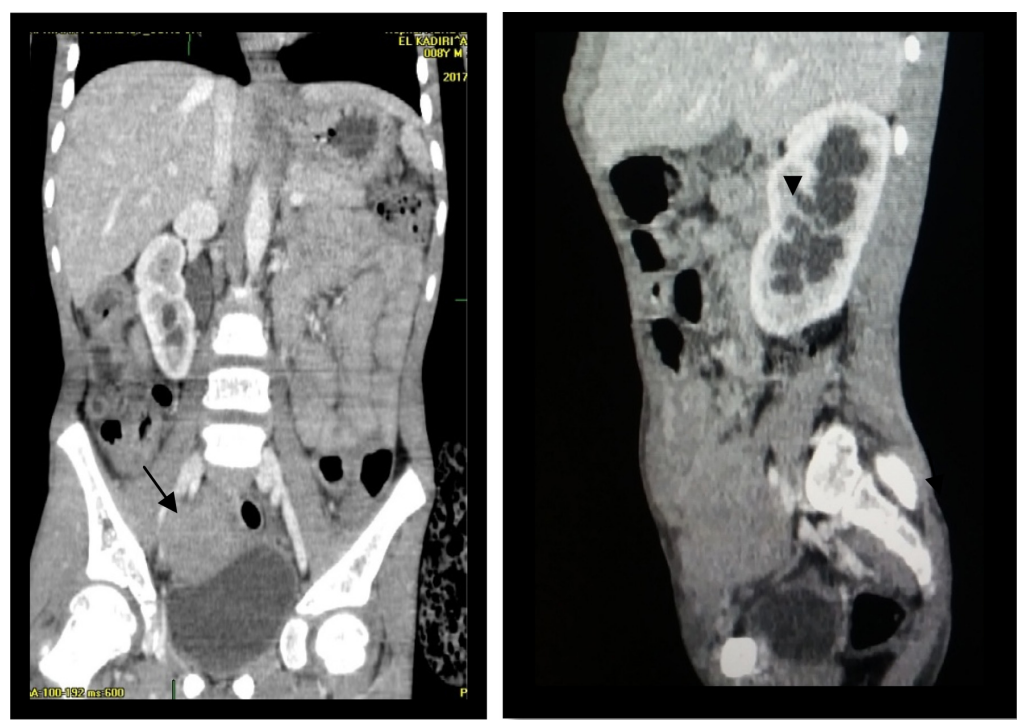

Figure 2. Axial and sagittal contrast-enhanced CT simages: right ureterohydronephrosis (arrow head) due to ureteral stenosis by the pelvic Infiltrative desmoid tumor (arrow). 


\section{Discussion}

Desmoid tumors are aggressive, deep fibromatosis occur in muscles and/or their sheaths and fasciae. It is a proliferation of fibroblastic tissue, infiltrating, non-metastatic but recurrent [1] [2].

The intra-abdominal form accounts for $5 \%$ to $7 \%$ of these tumors in children [3] [4]. The pelvic localization is a variety.

These desmoid tumors are frequently sporadic or may be associated with some genetic diseases such as familial adenomatous polyposis [5]. They can occur at any age in children with a peak between 5 years and 8 years [6]. The scarcity of intra-abdominal localization in children less than 10 years of age means that these tumors are underdiagnosed [1].

A female predominance was noted for pelvic localization [1]. The hypothesis of the link between tumor growth and endogenous estrogen levels seems not to be applicable in our case, since our patient is a male child. Clinically, these tumors progressively increase in size and can remain asymptomatic for a long time. They can be revealed by symptoms and complications of a mechanical nature (a hydroureteronephrosis, an occlusive syndrome, a deep vein thrombosis...).

Radiological exploration, based mainly on CT and MRI, makes it easy to discuss the diagnosis.

Ultrasound is usually done as a first intention imaging. It does not allow providing specific signs to make the diagnosis, but allows locating the tumor, which appears as a tissular mass hypoor hyper echogenic, well or poorly defined [7]; it allows appreciating its connections with neighboring organs, searching for other deep localizations and guiding a percutaneous puncture-biopsy. It is particularly useful for the early detection of tumor recurrence, and can be performed as well for the follow-up of the already operated patients [8].

$\mathrm{CT}$ is the test of choice to discuss the diagnosis of intra-abdominal desmoid tumors [2] [9]. It assesses tumor borders and connections better, looks for complications and shows differential diagnosis with other tumors. The CT appearance of desmoid tumors varies according to their composition. The lesion may appear hypo-, iso- or hyper-dense compared to muscular tissue, with or without an enhancement after injection of the contrast agent [9].

MRI remains the test of choice. It allows a more precise analysis of the lesion and a better study of the connections. It also makes it possible to differentiate post-therapeutic remaining from tumor recurrence [9]. The tumor appears as a generally infiltrating mass, with lobulated or sometimes irregular borders, in iso or homogeneous hyposignal T1 and often in hypersignal T2. The contrast enhancement is heterogeneous after gadolinium injection. The presence of hyposignal bands, related to collagen bundles, on all sequences is very characteristic [9].

Despite their benign character, these tumors behave aggressively locally with a high recurrence rate of approximately $25 \%$ - $65 \%$ [10]. This is the case of our pa- 
tient, where the tumor surrounded the right ureter. Therefore, a diameter greater than $10 \mathrm{~cm}$, an invasion of the small intestine and bilateral hydroureteronephrosis are factors of poor prognosis [9].

The diagnosis of certainty remains histological and immunohistochemical [1].

Treatment of desmoid tumors of the pelvis often requires a multidisciplinary approach [6]. It remains poorly defined in children. Surgery should be proposed as first-line for localized tumors for complete resection [6]. For so-called "stable" non-evolutionary and uncomplicated forms, simple monitoring can be done initially [6]. As for forms that are ineradicable or at high surgical risk, first-line chemotherapy should be discussed.

\section{Conclusion}

Desmoid tumors of the pelvis are very rare in children and their diagnosis should be considered in front any progressive pelvic mass. CT is the best of choice to suggest the diagnosis of these tumors [2] [9], but MRI allows better exploration and better pre- and postoperative analysis.

\section{Conflicts of Interest}

The authors do not declare any conflict of interest.

\section{References}

[1] Enzinger, F.M. and Weiss, S.W. (1995) Soft Tissue Tumours. 3rd Edition, Mosby-Year-Book, St Louis, Missouri.

[2] Montagliani, L. and Duverger, V. (2008) Les tumeurs desmoïdes. Journal De Chirurgie, 145, 20-26. https://doi.org/10.1016/S0021-7697(08)70288-6

[3] Faulkner, L.B., Hajdu, S.I., Kher, U., La Quaglia, M., Exelby, P.R., Heller, G. and Wollner, N. (1995) Pediatric Desmoid Tumor: Retrospective Analysis of 63 Cases. Journal of Clinical Oncology, 13, 2813-2818. https://doi.org/10.1200/JCO.1995.13.11.2813

[4] Meazza, C., Bisogno, G., Gronchi, A., Fiore, M., Cecchetto, G., Alaggio, R., Milano, G.M., Sanova, M., Carli, M. and Ferrari, A. (2010) Aggressive Fibromatosis in Children and Adolescents: The Italian Experience. Cancers, 116, 233-240.

[5] Shields, C.J., Winter, D.C., Kirwan, W.O. and Redmond, H.P. (2001) Desmoid Tumors. European Journal of Surgical Oncology, 27, 701-706.

https://doi.org/10.1053/ejso.2001.1169

[6] Honeyman, J.N. and La Quaglia, M.P. (2012) Desmoid Tumors in the Pediatric Population. Cancers, 4, 295-306. https://doi.org/10.3390/cancers4010295

[7] Casillas, J., Sais, G.J., Greve, J.L., Iparraguirre, M.C. and Morillo, G. (1991) Imaging of Intra- and Extraabdominal Desmoid Tumors. Radio Graphics, 11, 959-968. https://doi.org/10.1148/radiographics.11.6.1749859

[8] Wang, Y., Tang, J. and Luo, Y. (2008) Sonographic Diagnosis of Fibromatosis. Journal of Clinical Ultrasound, 36, 330-334. https://doi.org/10.1002/jcu.20483

[9] Cotte, E., Glehen, O., Monneuse, O., Cotton, F., et al. (2004) Tumeurs desmoïdes associées à la polypose adénomateuse familiale. Gastroenterologie Clinique Et Biologique, 28, 574-581. https://doi.org/10.1016/S0399-8320(04)95014-2 
[10] Burke, A.P., Sobin, L.H., Shekitka, K.M., Federspiel, B.H. and Helwig, E.B. (1990) Intra-Abdominal Fibromatosis: A Pathologic Analysis of 130 Tumors with Comparaison of Clinical Subgroups. American Journal of Surgical Pathology, 14, 335-341. https://doi.org/10.1097/00000478-199004000-00004 